\title{
Fetal Wound Healing Biomarkers
}

\author{
Fernanda Rodrigues Helmo, Juliana Reis Machado, \\ Camila Souza de Oliveira Guimarães, Vicente de Paula Antunes Teixeira, \\ Marlene Antônia dos Reis, and Rosana Rosa Miranda Corrêa
}

Discipline of General Pathology, Federal University of Triangulo Mineiro, Frei Paulino 30, 38025-180 Uberaba, MG, Brazil

Correspondence should be addressed to Rosana Rosa Miranda Corrêa; rosana@patge.uftm.edu.br

Received 30 June 2013; Revised 7 October 2013; Accepted 9 October 2013

Academic Editor: Ralf Lichtinghagen

Copyright (C) 2013 Fernanda Rodrigues Helmo et al. This is an open access article distributed under the Creative Commons Attribution License, which permits unrestricted use, distribution, and reproduction in any medium, provided the original work is properly cited.

\begin{abstract}
Fetal skin has the intrinsic capacity for wound healing, which is not correlated with the intrauterine environment. This intrinsic ability requires biochemical signals, which start at the cellular level and lead to secretion of transforming factors and expression of receptors, and specific markers that promote wound healing without scar formation. The mechanisms and molecular pathways of wound healing still need to be elucidated to achieve a complete understanding of this remodeling system. The aim of this paper is to discuss the main biomarkers involved in fetal skin wound healing as well as their respective mechanisms of action.
\end{abstract}

\section{The Human Skin}

The skin is the largest organ of the human body and is responsible for the maintenance of homeostasis, hemodynamic control, sensory reception, and innate and adaptive immunity. The skin is divided into two layers: the epidermis and the dermis. The epidermis originates from the ectoderm and it is formed by different cell types. The dermis is derived from the mesoderm and is rich in dense connective tissues [1].

During embryonic development, the epidermis changes from a single layer of ectodermal cells at 7-8 days of gestation into a stratified, keratinized epithelium at 22-24 weeks of pregnancy [2]. Formation of hair follicles starts in the eighth week, and in the 12th week, the development of embryonic fibroblasts is organized in networks of collagen fibers $[3,4]$.

Type I collagen is the main component of the extracellular matrix (ECM) $[5,6]$ and it confers tensile strength [6]. Type I and type III collagen fibers are present in the fetal skin, and dermal fibroblast populations exhibit greater type I collagen compared to type III collagen staining [5]. Subsequently, the production of elastin by human skin fibroblasts increased from 7-fold to 14-fold between 17 and 19 weeks of pregnancy, reaching the levels found in neonatal skin fibroblasts [7]. The elastic tissue contributes to the structure of the fetal dermis and increases in quantity and complexity during intrauterine development [7].

With the advancement of the pregnancy, the number of epidermal cell layers increases and the hair follicles and sweat glands complete their maturation [8]. Fetal skin development is completed 30 weeks after conception $[3,9]$.

The complex maturation of human skin during fetal development is achieved by the action of chemical mediators. The organization and function of this organ may be compromised by numerous diseases or secondary mechanisms that lead to the loss of tissue continuity.

The knowledge and understanding of the mechanisms of action of molecular markers involved in fetal skin wound healing may contribute to treatment and prevention of skin injuries.

Therefore, the aim of this review is to discuss the main biomarkers involved in fetal wound healing, which have been recently described in the literature. The articles discussed herein are part of the collection of the National Center for Biotechnology (PubMed) and of the Virtual Health Library (VHL). The selected papers addressed the topic of this paper, regardless of the year of publication. 


\section{Fetal Skin Wound Healing}

Skin wound healing is an organic response to tissue injury, which leads to an acute and local inflammatory reaction, fibroblast proliferation, and subsequent deposition of collagen and elastic fibers in the ECM. Moreover, it causes cellular proliferation that results in neoangiogenesis and reepithelialization. In adults particularly, the remodeling process fails in terms of tissue regeneration and excessive deposition of collagen fibers into a disorganized network [10-12]; this leads to scar formation.

In contrast, fetal skin wound healing triggers a regenerative response that preserves the architecture, organization, and function of the injured area. Until midpregnancy, specific mechanisms and pathways stimulate a rapid reepithelialization, the absence of an inflammatory response, the preservation of tissue architecture, and consequently the absence of scar tissue $[2,8]$ as compared to the wound healing that occurs at the end of the gestational period and during adulthood.

Intrauterine compounds such as sterile amniotic fluid that is rich in hyaluronic acid and growth factors are responsible for the high capacity of tissue remodeling [8, $12,13]$. However, several studies carried out with different experimental models demonstrated that this ability is due to tissue immaturity and poor cell differentiation $[10,14]$, which is observed at the beginning of intrauterine development [14, 15] and leads to regeneration of the dermal, neurovascular, and appendage architecture $[11,16]$.

Nevertheless, the mechanisms involved in fetal skin wound healing still need to be elucidated. Evidence suggests that maintenance of collagen matrix organization, inflammatory response, cellular mediators, expression of specific genes, and contribution of stem cells seem to be essential for tissue recovery [11].

Moreover, the existence of a complex and synchronized response triggered by metabolic pathways present in the cellular components, such as in ECM, has been observed in human skin, appendages, and the epidermis.

\section{Fetal Wound Healing Biomarkers}

Until the second trimester of pregnancy, wound healing is an intrinsic property of fetal skin that is not correlated with characteristics related to the intrauterine environment [14]. However, starting from the third quarter of fetal development, fetal wound healing leads to loss of hair follicles, deposition of dense collagen fibers, and an increased inflammatory response $[16,17]$.

The efficient process of skin remodeling seems to be related to the balanced action of metalloproteinases of the ECM, such as collagenases, gelatinase A, and stromelysin-1, which are responsible for the degradation of different types of collagen fibers, elastin, or proteoglycans, fibronectin, and laminin. Molecular studies showed that these enzymes are expressed by dermal cells and basal keratinocytes and in structures around the blood vessels of fetal and adult skin, although they are not abundant in the latter. Therefore, the expression of these proteases would enable ECM renewal, epithelization, and angiogenesis [18].

Studies describing the mechanism of action of these enzymes in wound healing indicate that in early fetal skin development, gelatinase A and B have low activities. In advanced gestational age, an increased expression of these enzymes was observed in epidermal cells, fibroblasts, endothelial cells, and keratinocytes. Membrane type 1 is widely expressed in epithelial cell membranes and cytoplasm by the 13th week of development, whereas it is expressed in fibroblasts and epithelial cells in late pregnancy. The same pattern of expression was also observed with inhibitors of matrix metalloproteinases TIMP-2 and TIMP-1, as with the respective inhibitors of gelatinase A (MMP-2) and B (MMP9 ), weakly expressed during early intrauterine development [19].

In turn, lysyl oxidase enzyme is responsible for the harmony and connection of the collagen network in the ECM. The expression of this enzyme in fetal skin is 2-fold higher than that in adult skin. The catalytic power of lysyl oxidase in fetal fibroblasts is up to 1.7-fold higher than that in postnatal fibroblasts [20]. Depending on the pregnancy period, the differences in the expression of these enzymes may be related to deposition of a different set of collagen fibers in the cellular matrix.

Collagen is the most abundant component of the ECM; it provides tensile strength and modulates cell proliferation and migration during wound healing. Moreover, it shows a different deposition pattern in fetal and adult skin. In agreement with this observation, a molecular study was performed to assess the differences in the inhibition of fibroblast contraction. In response to $1 \mu \mathrm{M}$ prostaglandin E2 (PGE2), significant contraction inhibition, 55\% and 33\% upon $2 \mathrm{~h}$ and $4 \mathrm{~h}$ stimulations, respectively, was achieved in adult skin. However, in fetal skin assessed at the same time intervals, the inhibition was only $27 \%$ and $22 \%$, respectively. Moreover, fibroblast distribution was also reduced in adult skin $4 \mathrm{~h}$ after the onset of PGE2 action, whereas in fetal skin, this characteristic was not modified. Interestingly, a different mechanism for regulation of actin cytoskeleton likely exists in fetal and adult fibroblasts, since distinct morphology and expression patterns of agonist receptors were also observed in these cells [21].

Migration of fetal fibroblasts is more rapid, depending upon the time after the injury. In agreement with experimental measurements, the action of PGE2 quantitatively differs between fetal and adult fibroblasts, although it remains qualitatively similar. This means that when exposed to maximum PGE2 concentration, adult fibroblasts lose up to $80 \%$ of their ability to migrate when compared to fetal fibroblasts, in which the loss of migration is less than $60 \%$ [22]. Presumably, this effect contributes to fetal skin repair in the presence of lower inflammatory response at the site of injury.

In turn, cyclooxygenase-2 (COX-2) and its major metabolite, PGE2, lead to fibrotic scar formation in fetal skin. Studies reveal that, $24 \mathrm{~h}$ after injury, COX-2 messenger ribonucleic acid (mRNA) and protein expression in basal keratinocytes and immune and stromal cells, as well as PGE2 levels, were significantly higher in fetal mouse skin after 
18 days of pregnancy when compared to 15 days of pregnancy, in which this response was absent. Therefore, this suggests that COX-2 should modulate regeneration of the healing tissue, as increased tissue exposure to PGE2 seems to delay the reepithelialization, resulting in scar formation [16].

Similarly, interleukins lead to progression of the inflammatory response and have been identified as important mediators of postnatal tissue healing. As an example, interleukin6 (IL-6) stimulates the proliferation and migration of keratinocytes, in addition to promoting angiogenesis in adult skin healing. By comparative molecular analyses of fetal and adult fibroblasts with respect to IL-6 synthesis, lower production of this mediator was observed in fetal fibroblasts. In contrast, adult fibroblasts synthesize a greater amount of IL-6 mRNA compared to fetal fibroblasts [23].

A similar expression in the inflammatory cascade is observed for interleukin-8 (IL-8), which is significantly induced in adult fibroblasts in response to lower concentrations of platelet-derived growth factor (PDGF). Therefore, expression of IL-8 mRNA is significantly lower in fetal fibroblasts [24].

To support these scientific findings, an experimental transgenic model was created, in which IL-6 was secreted in the absence of interleukin-10 (IL-10) in response to any kind of skin injury compared to control mice. The results showed an increased and abnormal deposition of collagen fibers, loss of dermal appendages, scar formation in the fetal skin of transgenic mice, and infiltration of inflammatory cells demonstrated by expression of the CD-45 marker. In control mice, skin remodeling occurred with minimal infiltration of inflammatory cells, with normal collagen distribution and recovery of dermal structure and its components [25].

These results demonstrate the possible correlation between increased IL- 6 and IL- 8 production and the prevalence of an inflammatory response in adult skin, which favors scar formation. Contrarily, increased IL-10 production is antiinflammatory, as it diminishes the recruitment of inflammatory cells during fetal wound healing [25].

Studies demonstrated that transforming growth factor$\beta$ (TGF- $\beta$ ) is involved in wound healing by promoting fibroblast differentiation and ECM remodeling [26, 27]. TGF$\beta$ is a potent chemoattractant of macrophages, neutrophils, and fibroblasts, stimulates extracellular matrix synthesis, and prevents its degradation by upregulating the expression of tissue inhibitors of metalloproteinases (TIMPs) and downregulating the expression of proteases [28].

It is known that TGF- $\beta 1$ and TGF- $\beta 3$ are significantly expressed in human fetal and adult skin, respectively $[26,29$, 30]. However, TGF- $\beta 1$ and TGF- $\beta 2$ receptors are expressed in both fetal and adult dermis tissues. In addition to establishing a correlation between greater predisposition to developing scars $[26,29]$ and the differences in these signaling pathways, it is also possible to infer the existence of an ideal concentration of TGF- $\beta 1$ in fetal skin, which may provide beneficial effects for the complete regeneration of the tissue [26].

Fetal studies in mice demonstrated that the peak action of TGF- $\beta 1$ was achieved in the ECM up to $24 \mathrm{~h}$ after the onset of injury and in dermal cells after $12 \mathrm{~h}$, thus returning to basal level $36 \mathrm{~h}$ after the lesion. However, with increased gestational age, TGF- $\beta 1$ was produced more than $48 \mathrm{~h}$ after injury. Therefore, it was observed that the time of action of TGF- $\beta 1$ was proportional to the intensity of the inflammatory cell infiltration at the site of injury [27].

Expression of TGF- $\beta 3$ was already significant at 24 to $36 \mathrm{~h}$ after the injury at the beginning of the pregnancy. However, with the increased gestational age, the peaks were synchronized every $24 \mathrm{~h}$ and concentrated for $72 \mathrm{~h}$ in the basal layer of the epidermis. In the ECM and dermal cells, increased TGF- $\beta 3$ was also detected up to $36 \mathrm{~h}$ after skin injury, whereas in fetal dermis and with increased gestational age, TGF- $\beta 3$ presented the same pattern of expression as the epidermis [27].

In another study assessing skin fragments derived from human donors of different ages, TGF- $\beta 1$ inhibited the synthesis of the genetic material of fetal fibroblasts in vitro, although the opposite effect was found in adults fibroblasts. Moreover, when coupled with the PDGF-BB isoform, the latter inhibited the action of TGF- $\beta 1$ in fetal fibroblasts, although it did enhance the action of TGF- $\beta 1$ in adult fibroblasts. In summary, TGF- $\beta 1$ inhibits the proliferation of these cells in the fetal ECM [31].

Hydrogen peroxide and products released by phagocytic interferes with scarless healing, possibly through the induction of TGF- $\beta 1$. Hydrogen peroxide also increased the proliferation of fetal fibroblasts, which could contribute to an increase in the fibrosis [17].

Detection of differential expression of fetal mouse fibroblast receptors suggested a correlation between different patterns of tyrosine phosphorylation in fetal and adult cells. Epidermal growth factor receptors, namely, discoidin domain receptor 1 (DDR1) and Shc, are found in embryos and are related to rapid reepithelialization, organization of collagen fibers, and architecture regeneration of the injured tissue [32]. $\mathrm{Shc}$ is an $\mathrm{SH} 2$ domain-containing protein that is tyrosine phosphorylated in response to a variety of growth factors [33]. Shc becomes tyrosine phosphorylated upon stimulation with a number of growth factors including epidermal growth factor EGF [34].

Molecular studies of genetic patterns involved in fetal and postnatal wounds healing during three different periods identified expression of 321, 216, and 27 genes at 1, 12, and $24 \mathrm{~h}$ after the onset of skin injury, respectively. In fetal skin, intense gene transcription was observed up to $12 \mathrm{~h}$, followed by a subsequent decrease after the first $24 \mathrm{~h}$. This supports the rapidity of the healing process. The gene transcription factors CP2-like-2, Grainyhead-like epithelial transactivator, and retinoblastoma modulator exhibited this pattern, as well as the genes responsible for cell proliferation, such as Janus Kinase-2 and the tumor differentially expressed-2 gene. Expression of other genes such as transcription factors of immediate-early response- 3 and growth response protein1 decreased after $24 \mathrm{~h}$. In the postnatal period, three genes (secretory proteoglycans granule, chemokine-12, and CD63) correlated with the formation of the fibrotic tissue [35].

Expression of specific RNA fragments, namely, microRNAs, has been identified as potential key differentiators and initiators of the wound healing response during fetal and postnatal development. Studies carried out with fragments derived from human tissue showed that RNA, namely, 
microR-29b, microR-29c, and microR-192, correlated with modulation of different proteins in the ECM and signaling pathways involved in skin remodeling [36].

Another important molecular aspect refers to the apoptotic response mediated by caspase-7 at the beginning of the pregnancy that seems to be responsible for the inactivation and cleavage of poly-ADP ribose polymerase (PARP) and the consequent programmed removal of damaged cells. By assessing this mechanism, reduced expression of caspase- 3 and increased activation of the Akt marker were observed in hypertrophic lesions, an effect that induced a greater number of apoptotic fibroblasts. In mice, during early fetal skin development, the protease action of caspase-7 was 2-fold higher than during tissue remodeling, an effect that was also observed for cleaved PARP. In contrast, no variations were observed or reduced at the end of the pregnancy period in mice [37].

Among the proteins of the cellular cytoskeleton, suppression of Flightless-I (FLii), a member of the remodeling actins family, plays an important role in injury contraction. Analyses carried out using fetal mouse skin showed that, in the presence of an injury, FLii temporarily translocates from the cytoplasm to the nucleus of those keratinocytes distant from the wound bed. This favors cell cycling and reduces the injury up to $92 \%$ of the initial area $48 \mathrm{~h}$ later. However, increased expression of FLii during pregnancy enables less than $25 \%$ of injury reduction [38], suggesting that lower FLii expression favors tissue remodeling.

HASA (hyaluronan-stimulating activity), a common fetal glycoprotein mediating wound healing, promotes fibroblast movement through the ECM and is nearly absent in adult wound healing [28]. Hyaluronan (HA) is a macromolecule synthesized in fibroblasts and is inhibited by a concentration of TGF- $\beta 1$ lower than $0.1 \mathrm{ng} / \mathrm{mL}$. Moreover, HA expression is related to the cellular density of fibroblasts at the site of injury [39].

When assessing the regeneration of fragments of fetal epidermis that were cultured and subjected to heat shock, induced expressions of protein keratin-17 (K17), skin-derived antileucoproteinase (SKALP), and keratin-14 (K14) were observed 21 days later. Compared to adult skin, only K17 was expressed by culture fragments, whereas K14 and SKALP were detected in the regenerating epidermis $[2,8]$. Therefore, it is possible to correlate these markers with the epidermal reepithelialization signaling pathway activated upon fetal skin wound healing.

In the dermis, expression of chondroitin sulfate (CS) was already observed in both fetal and adult skin during the same period of time [2]. However, in fetal skin, this marker was detected in the papillary dermis and in the upper region of the reticular dermis in the 16th week of embryonic development. In the adult dermis, SC was identified only in the basal membrane of the dermal-epidermal junction and blood vessels [8]. This demonstrated that ECM renewal capacity relies on the expression of these proteins, particularly when its expression is compared after injury among different fragments.

Proliferation of fetal skin is associated with the presence of keratin-i67 in the basal layer of the epidermis and dermis.
Between the 13th and 14th weeks of pregnancy, expression of keratin-i67 is significantly higher when compared to levels obtained in the 16th to 22nd weeks and to both layers of adult skin. Epidermal differentiation enables identification of the presence of keratin-10 and involucrin in the intermediate layers, as well as primordial hair follicle infundibulum between the 13th and 14th weeks. Stratification is coupled to the expression of K14 in the epidermal basal layer from the 13th week, in hair follicles and the basal layer of the sebaceous glands starting from the 16th week of development [2].

A transmembrane glycoprotein, integrin, acts as a receptor for ECM components and favors adhesion, migration, proliferation, and cell differentiation. Among the most important components are the fibronectin receptors $(\alpha 5 \beta 1$, $\alpha \mathrm{v} \beta 3$, and $\alpha 3 \beta 1)$, fibronectin and tenascin ( $\alpha \mathrm{v} 5 \beta 6)$, collagen $(\alpha 2 \beta 1$ and $\alpha 3 \beta 1)$, laminin, and collagen type IV ( $\alpha 6 \beta 4$ and $\alpha 6 \beta 1$ ). Studies on fetal skin injuries demonstrate high expression of collagen receptors $(\alpha 2)$ and collagen/fibronectin $(\alpha 3)$ in the pericellular region of epidermal keratinocytes and hair follicles, whereas laminin ( $\alpha 3$ and $\beta 4)$ has been found, in particular, in the pericellular region of the epidermal basal layer [40].

This observation may be confirmed by tenascin expression that is high at early stages in the epidermal-dermal junction. Subsequently, tenascin increases throughout the fetal dermis $8 \mathrm{~h}$ after the onset of the injury [41]. In adult skin, a higher expression was observed $24 \mathrm{~h}$ after injury [ 42 , 43], suggesting that the rapid involvement of this protein contributes to an efficient and effective epithelization of the skin.

\section{Conclusion}

The mechanisms and molecular pathways of wound healing still need to be elucidated to understand skin remodeling. Until the second trimester, fetal skin maintains specific properties that lead to a complete recovery of tissue architecture, which includes the epidermis, the dermis, and skin appendages. This intrinsic capacity requires that biochemical components start signaling at the cellular level and culminate with the secretion of transforming factors and expression of specific receptors and markers that promote wound healing without scar formation.

Finally, the discovery and understanding of the functions of these biomarkers are essential to understand fetal healing and, consequently, to prevent and treat skin injuries.

\section{References}

[1] L. R. Souto, J. Rehder, J. Vassallo, M. L. Cintra, M. H. Kraemer, and M. B. Puzzi, "Model for human skin reconstructed in vitro composed of associated dermis and epidermis," Sao Paulo Medical Journal, vol. 124, no. 2, pp. 71-76, 2006.

[2] N. A. Coolen, K. C. W. M. Schouten, E. Middelkoop, and M. M. W. Ulrich, "Comparison between human fetal and adult skin," Archives of Dermatological Research, vol. 302, no. 1, pp. 47-55, 2010. 
[3] L. De Noronha, F. Medeiros, V. D. Martins et al., "Malformations of the central nervous system: analysis of 157 pediatric autopsics," Arquivos de Neuro-Psiquiatria, vol. 58, no. 3 B, pp. 890896, 2000.

[4] F. Serri, W. Montagna, and H. Mescon, "Studies of the skin of the fetus and the child. II. glycogen and amylophos-phorylase in the skin of the fetus," The Journal of Investigative Dermatology, vol. 39, pp. 199-217, 1962.

[5] H. E. Brink, S. S. Stalling, and S. B. Nicoll, "Influence of serum on adult and fetal dermal fibroblast migration, adhesion, and collagen expression," In Vitro Cellular and Developmental Biology-Animal, vol. 41, no. 8-9, pp. 252-257, 2005.

[6] L. Cuttle, M. Nataatmadja, J. F. Fraser, M. Kempf, R. M. Kimble, and M. T. Hayes, "Collagen in the scarless fetal skin wound: detection with picrosirius-polarization," Wound Repair and Regeneration, vol. 13, no. 2, pp. 198-204, 2005.

[7] G. C. Sephel, A. Buckley, and J. M. Davidson, "Developmental initiation of elastin gene expression by human fetal skin fibroblasts," Journal of Investigative Dermatology, vol. 88, no. 6, pp. 732-735, 1987.

[8] N. A. Coolen, K. C. Schouten, B. K. Boekema, E. Middelkoop, and M. M. Ulrich, "Wound healing in a fetal, adult, and scar tissue model: a comparative study," Wound Repair and Regeneration, vol. 18, no. 3, pp. 291-301, 2010.

[9] J. Ersch and T. Stallmach, "Assessing gestational age from histology of fetal skin: an autopsy study of 379 fetuses," Obstetrics and Gynecology, vol. 94, no. 5, pp. 753-757, 1999.

[10] D. L. Cass, K. M. Bullard, K. G. Sylvester, E. Y. Yang, M. T. Longaker, and N. S. Adzick, "Wound size and gestational age modulate scar formation in fetal wound repair," Journal of Pediatric Surgery, vol. 32, no. 3, pp. 411-415, 1997.

[11] B. J. Larson, M. T. Longaker, and H. P. Lorenz, "Scarless fetal wound healing: a basic science review," Plastic and Reconstructive Surgery, vol. 126, no. 4, pp. 1172-1180, 2010.

[12] H. P. Lorenz, D. J. Whitby, M. T. Longaker, and N. S. Adzick, "Fetal wound healing: the ontogeny of scar formation in the non-human primate," Annals of Surgery, vol. 217, no. 4, pp. 391396, 1993.

[13] C. Dang, K. Ting, C. Soo, M. T. Longaker, and H. P. Lorenz, "Fetal wound healing current perspectives," Clinics in Plastic Surgery, vol. 30, no. 1, pp. 13-23, 2003.

[14] J. R. Armstrong and M. W. Ferguson, "Ontogeny of the skin and the transition from scar-free to scarring phenotype during wound healing in the pouch young of a marsupial, Monodelphis domestica," Developmental Biology, vol. 169, no. 1, pp. 242-260, 1995.

[15] A. Leung, T. M. Crombleholme, and S. G. Keswani, "Fetal wound healing: implications for minimal scar formation," Current Opinion in Pediatrics, vol. 24, no. 3, pp. 371-378, 2012.

[16] T. A. Wilgus, V. K. Bergdall, K. L. Tober et al., "The impact of cyclooxygenase-2 mediated inflammation on scarless fetal wound healing," American Journal of Pathology, vol. 165, no. 3 , pp. 753-761, 2004.

[17] T. A. Wilgus, V. K. Bergdall, L. A. Dipietro, and T. M. Oberyszyn, "Hydrogen peroxide disrupts scarless fetal wound repair," Wound Repair and Regeneration, vol. 13, no. 5, pp. 513519, 2005.

[18] K. M. Bullard, D. L. Cass, M. J. Banda, and N. S. Adzick, "Transforming growth factor beta-1 decreases interstitial collagenase in healing human fetal skin," Journal of Pediatric Surgery, vol. 32, no. 7, pp. 1023-1027, 1997.
[19] W. Chen, X. Fu, S. Ge, T. Sun, and Z. Sheng, "Differential expression of matrix metalloproteinases and tissue-derived inhibitors of metalloproteinase in fetal and adult skins," International Journal of Biochemistry and Cell Biology, vol. 39, no. 5, pp. 9971005, 2007.

[20] A. S. Colwell, T. M. Krummel, M. T. Longaker, and H. P. Lorenz, "Wnt-4 expression is increased in fibroblasts after TGFbetal stimulation and during fetal and postnatal wound repair," Plastic and Reconstructive Surgery, vol. 117, no. 7, pp. 2297-2301, 2006.

[21] A. Parekh, V. C. Sandulache, A. S. Lieb, J. E. Dohar, and P. A. Hebda, "Differential regulation of free-floating collagen gel contraction by human fetal and adult dermal fibroblasts in response to prostaglandin E2 mediated by an EP2/cAMPdependent mechanism," Wound Repair and Regeneration, vol. 15, no. 3, pp. 390-398, 2007.

[22] V. C. Sandulache, A. Parekh, H.-S. Li-Korotky, J. E. Dohar, and P. A. Hebda, "Prostaglandin E2 differentially modulates human fetal and adult dermal fibroblast migration and contraction: implication for wound healing," Wound Repair and Regeneration, vol. 14, no. 5, pp. 633-643, 2006.

[23] K. W. Liechty, N. S. Adzick, and T. M. Crombleholme, "Diminished interleukin 6 (IL-6) production during scarless human fetal wound repair," Cytokine, vol. 12, no. 6, pp. 671-676, 2000.

[24] K. W. Liechty, T. M. Crombleholme, D. L. Cass, B. Martin, and N. S. Adzick, "Diminished interleukin-8 (IL-8) production in the fetal wound healing response," Journal of Surgical Research, vol. 77, no. 1, pp. 80-84, 1998.

[25] K. W. Liechty, H. B. Kim, N. S. Adzick, and T. M. Crombleholme, "Fetal wound repair results in scar formation in interleukin10 -deficient mice in a syngeneic murine model of scarless fetal wound repair," Journal of Pediatric Surgery, vol. 35, no. 6, pp. 866-873, 2000.

[26] S. R. Goldberg, R. P. McKinstry, V. Sykes, and D. A. Lanning, "Rapid closure of midgestational excisional wounds in a fetal mouse model is associated with altered transforming growth factor-beta isoform and receptor expression," Journal of Pediatric Surgery, vol. 42, no. 6, pp. 966-971, 2007.

[27] C. Soo, S. R. Beanes, F.-Y. Hu et al., "Ontogenetic transition in fetal wound transforming growth factor-beta regulation correlates with collagen organization," American Journal of Pathology, vol. 163, no. 6, pp. 2459-2476, 2003.

[28] M. R. Namazi, M. K. Fallahzadeh, and R. A. Schwartz, "Strategies for prevention of scars: what can we learn from fetal skin?" International Journal of Dermatology, vol. 50, no. 1, pp. 85-93, 2011.

[29] K. M. Sullivan, H. P. Lorenz, M. Meuli, R. Y. Lin, and N. S. Adzick, "A model of scarless human fetal wound repair is deficient in transforming growth factor beta," Journal of Pediatric Surgery, vol. 30, no. 2, pp. 198-203, 1995.

[30] A. S. Colwell, T. M. Krummel, M. T. Longaker, and H. P. Lorenz, "Fetal and adult fibroblasts have similar TGF-betamediated, smad-dependent signaling pathways," Plastic and Reconstructive Surgery, vol. 117, no. 7, pp. 2277-2283, 2006.

[31] H. Pratsinis, C. C. Giannouli, I. Zervolea, S. Psarras, D. Stathakos, and D. Kletsas, "Differential proliferative response of fetal and adult human skin fibroblasts to transforming growth factor-beta," Wound Repair and Regeneration, vol. 12, no. 3, pp. 374-383, 2004.

[32] G. S. Chin, W. J. Kim, T. Y. Lee et al., "Differential expression of receptor tyrosine kinases and Shc in fetal and adult rat fibroblasts: toward defining scarless versus scarring fibroblast 
phenotypes," Plastic and Reconstructive Surgery, vol. 105, no. 3, pp. 972-979, 2000.

[33] A. G. Batzer, D. Rotin, J. M. Urena, E. Y. Skolnik, and J. Schlessinger, "Hierarchy of binding sites for Grb2 and Shc on the epidermal growth factor receptor," Molecular and Cellular Biology, vol. 14, no. 8, pp. 5192-5201, 1994.

[34] A. Hashimoto, M. Kurosaki, N. Gotoh, M. Shibuya, and T. Kurosaki, "Shc regulates epidermal growth factor-induced activation of the JNK signaling pathway," Journal of Biological Chemistry, vol. 274, no. 29, pp. 20139-20143, 1999.

[35] A. S. Colwell, M. T. Longaker, and H. P. Lorenz, "Identification of differentially regulated genes in fetal wounds during regenerative repair," Wound Repair and Regeneration, vol. 16, no. 3, pp. 450-459, 2008.

[36] J. Cheng, H. Yu, S. Deng, and G. Shen, "MicroRNA profiling in mid- and late-gestational fetal skin: implication for scarless wound healing," The Tohoku Journal of Experimental Medicine, vol. 221, no. 3, pp. 203-209, 2010.

[37] R. Carter, V. Sykes, and D. Lanning, "Scarless fetal mouse wound healing may initiate apoptosis through caspase 7 and cleavage of PARP," Journal of Surgical Research, vol. 156, no. 1, pp. 74-79, 2009.

[38] C.-H. Lin, J. M. Waters, B. C. Powell, R. M. Arkell, and A. J. Cowin, "Decreased expression of Flightless I, a gelsolin family member and developmental regulator, in early-gestation fetal wounds improves healing," Mammalian Genome, vol. 22, no. 56, pp. 341-352, 2011.

[39] I. R. Ellis and S. L. Schor, "Differential effects of TGF-betal on hyaluronan synthesis by fetal and adult skin fibroblasts: implications for cell migration and wound healing," Experimental Cell Research, vol. 228, no. 2, pp. 326-333, 1996.

[40] D. L. Cass, K. M. Bullard, K. G. Sylvester et al., "Epidermal integrin expression is upregulated rapidly in human fetal wound repair," Journal of Pediatric Surgery, vol. 33, no. 2, pp. 312-316, 1998.

[41] D. J. Whitby, M. T. Longaker, M. R. Harrison, N. S. Adzick, and M. W. Ferguson, "Rapid epithelialisation of fetal wounds is associated with the early deposition of tenascin," Journal of Cell Science, vol. 99, no. 3, pp. 583-586, 1991.

[42] K. M. Bullard, M. T. Longaker, and H. P. Lorenz, "Fetal wound healing: current biology," World Journal of Surgery, vol. 27, no. 1, pp. 54-61, 2003.

[43] M. T. Longaker, D. J. Whitby, N. S. Adzick, L. B. Kaban, and M. R. Harrison, "Fetal surgery for cleft lip: a plea for caution," Plastic and Reconstructive Surgery, vol. 88, no. 6, pp. 1087-1092, 1991. 


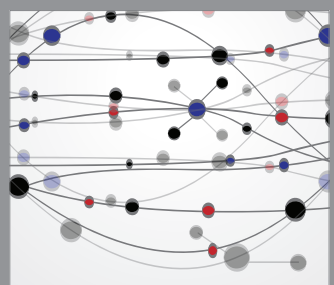

The Scientific World Journal
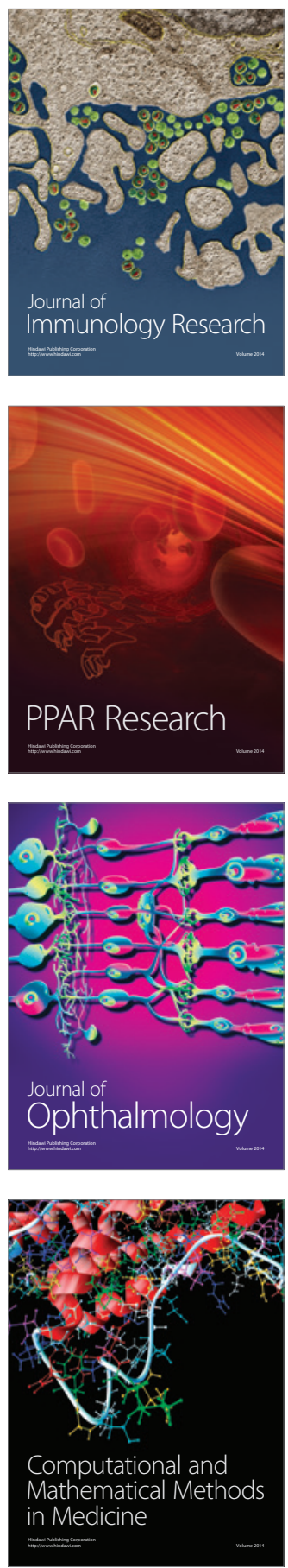

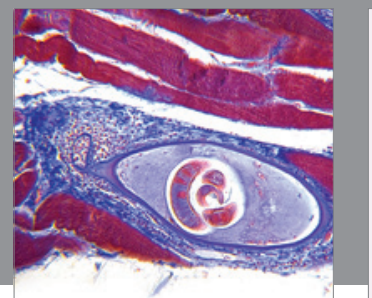

Gastroenterology

Research and Practice
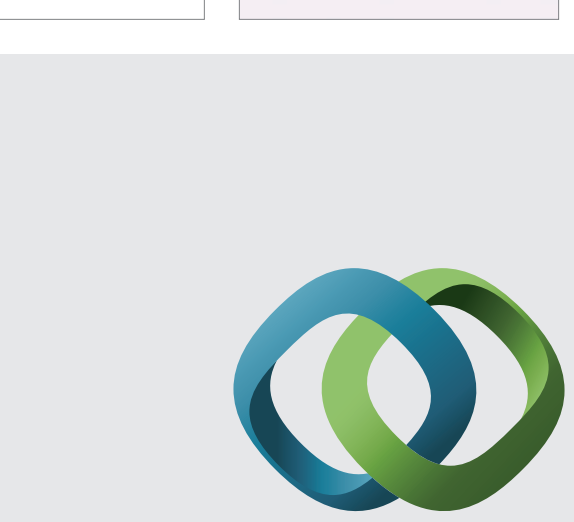

\section{Hindawi}

Submit your manuscripts at

http://www.hindawi.com
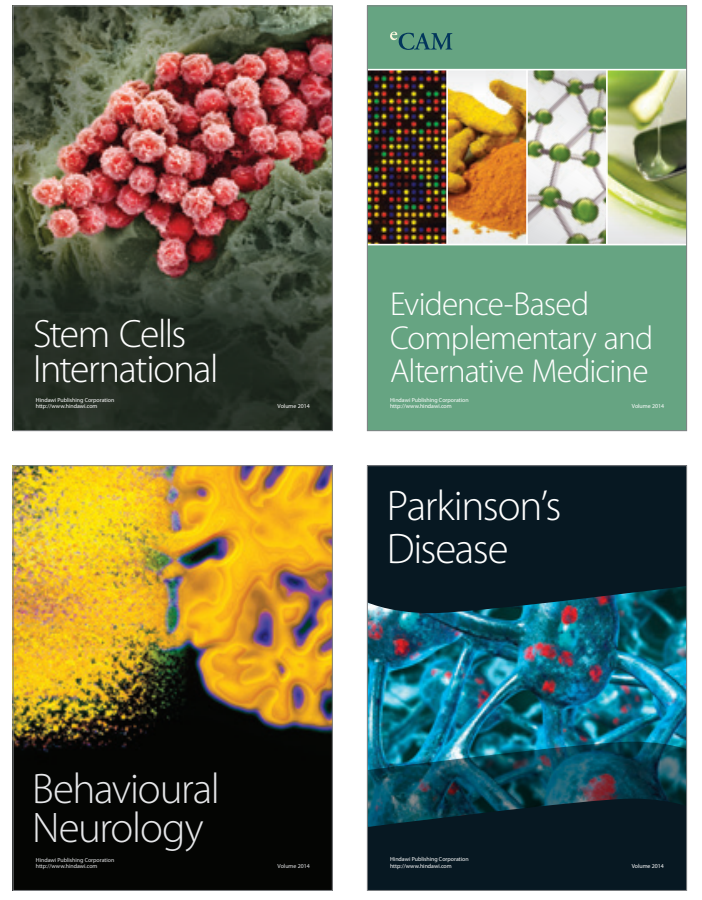
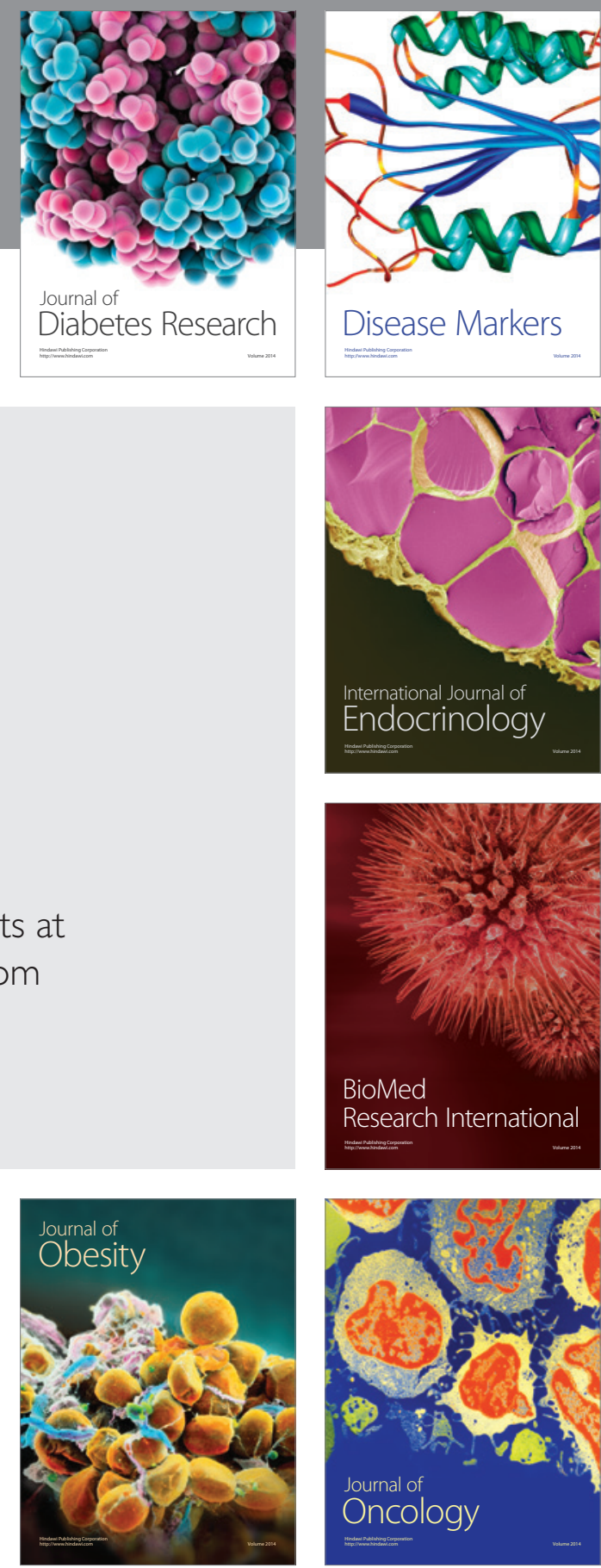

Disease Markers
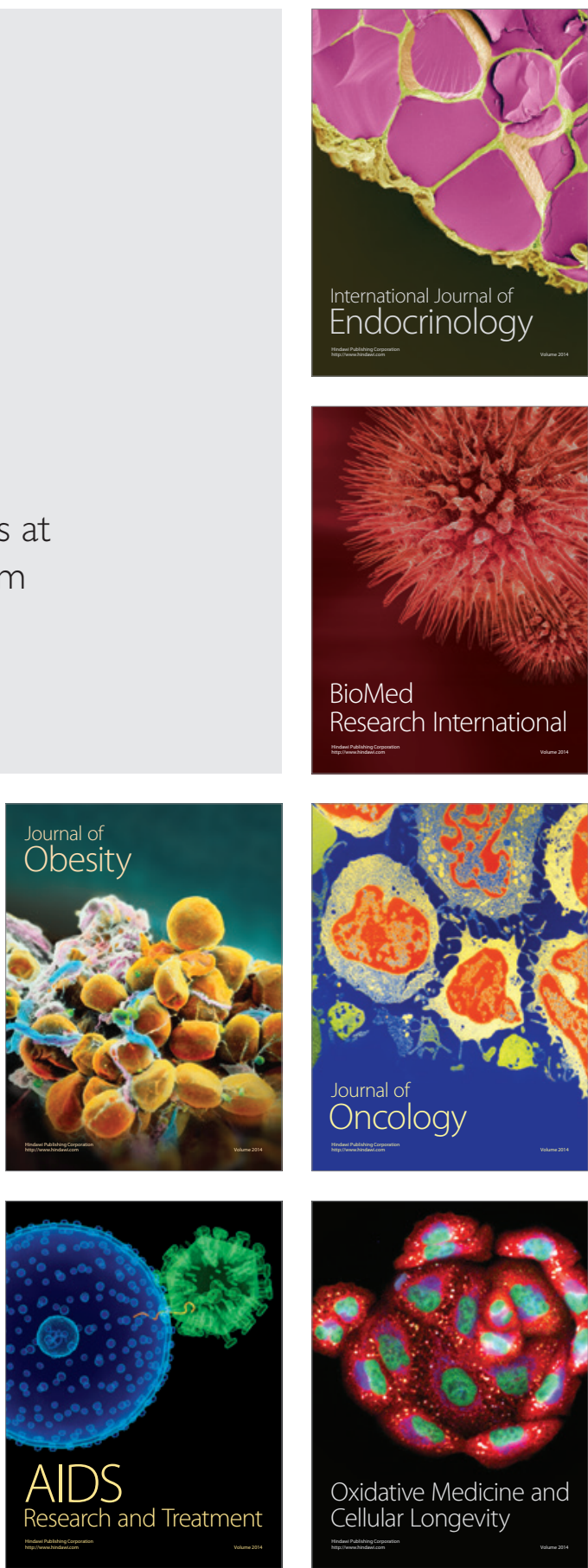